ACCEPTED MANUSCRIPT

\title{
Influence of photo-generated carriers on current spreading in double diode structures for electroluminescent cooling
}

To cite this article before publication: Ivan Radevici et al 2018 Semicond. Sci. Technol. in press https://doi.org/10.1088/1361-6641/aab6c3

\section{Manuscript version: Accepted Manuscript}

Accepted Manuscript is "the version of the article accepted for publication including all changes made as a result of the peer review process, and which may also include the addition to the article by IOP Publishing of a header, an article ID, a cover sheet and/or an 'Accepted

Manuscript' watermark, but excluding any other editing, typesetting or other changes made by IOP Publishing and/or its licensors"

This Accepted Manuscript is @ 2018 IOP Publishing Ltd.

During the embargo period (the 12 month period from the publication of the Version of Record of this article), the Accepted Manuscript is fully protected by copyright and cannot be reused or reposted elsewhere.

As the Version of Record of this article is going to be / has been published on a subscription basis, this Accepted Manuscript is available for reuse under a CC BY-NC-ND 3.0 licence after the 12 month embargo period.

After the embargo period, everyone is permitted to use copy and redistribute this article for non-commercial purposes only, provided that they adhere to all the terms of the licence https://creativecommons.org/licences/by-nc-nd/3.0

Although reasonable endeavours have been taken to obtain all necessary permissions from third parties to include their copyrighted content within this article, their full citation and copyright line may not be present in this Accepted Manuscript version. Before using any content from this article, please refer to the Version of Record on IOPscience once published for full citation and copyright details, as permissions will likely be required. All third party content is fully copyright protected, unless specifically stated otherwise in the figure caption in the Version of Record.

View the article online for updates and enhancements. 


\title{
Influence of photo-generated carriers on current spreading in double diode structures for electroluminescent cooling
}

\author{
Ivan Radevicił, Jonna Tiira, Toufik Sadi and Jani Oksanen \\ E-mail: ivan.radevici@aalto.fi \\ Engineered Nanosystems Group, Aalto University, Aalto 00076, Finland \\ 2 March 2018
}

Abstract. Current crowding close to electrical contacts is a common challenge in all optoelectronic devices containing thin current spreading layers (CSLs). We analyze the effects of current spreading on the operation of the so-called double diode structure (DDS), consisting of a light emitting diode (LED) and a photodiode (PD) fabricated within the same epitaxial growth process, and providing an attractive platform for studying electroluminescent (EL) cooling under high bias conditions. We show that current spreading in the common n-type layer between the LED and the PD can be dramatically improved by the strong optical coupling between the diodes, as the coupling enables a photo-generated current through the PD. This reduces the current in the DDS CSL and enables studying EL cooling using structures that are not limited by the conventional light extraction challenges encountered in normal LEDs. The current spreading in the structures is studied using optical imaging techniques, electrical measurements, simulations, as well as simple equivalent circuit models developed for this purpose. The improved current spreading leads further to a mutual dependence with the coupling efficiency, which is expected to facilitate the process of optimizing the DDS. We also report a new improved value of $63 \%$ for the DDS coupling quantum efficiency (CQE).

Keywords: electroluminescent cooling, quantum efficiency, radiative and non-radiative recombination, III-V semiconductors, double diode structures, current spreading Submitted to: Semicond. Sci. Technol. 
Electroluminescent (EL) refrigeration was theoretically anticipated almost 60 years ago $[1,2]$, but experimentally it still remains more of a curiosity rather 35 than a well acknowledged possibility. Fundamentally,

5 EL cooling is based on simple thermodynamics of light emitting diodes (LEDs), allowing a diode to emit a photon with an energy larger than the bandgap of the semiconductor material the LED is made of. This ap-40 plies even when the bias voltage of the LED is arbi10 trarily small $[3-5]$.

As in the case of photoluminescent (PL) cooling, the 'missing' energy is extracted from heat obtained from the phonons in the crystal lattice [6]. For a45 sufficiently high external quantum efficiency (EQE) or

15 low bias voltage the absorbed heat can overcome the internal heat generation, resulting in refrigeration of the LED $[4,6-8]$.

In the last years, the interest in the EL cooling 50 has started to increase and the field has gained 20 wider and more systematic attention [7,9-12]. Up to date, however, there are no firm experimental demonstrations of EL cooling beyond the small bias regime, where the LED bias is only a small fraction 55 of the voltage corresponding to the materials' bandgap 25 and the EQE and cooling power are extremely small $[4,8]$. Under the high bias condition where the EQE is high and the bias is only slightly below the voltage corresponding to the semiconductor bandgap, it is 60 still necessary to further improve the light emission 30 efficiency to reach the cooling regime.

One of the most obvious obstacles that strongly contributes to the efficiency of LEDs and the challenge

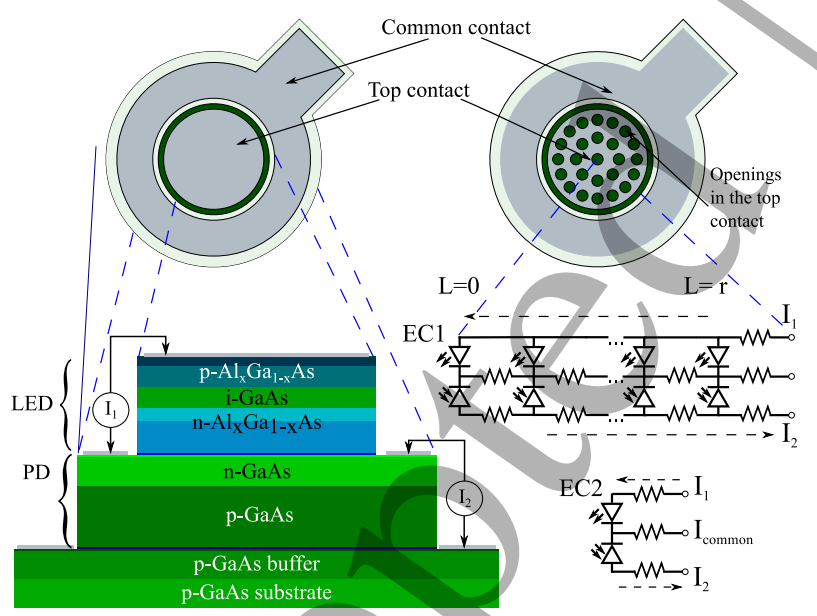

Figure 1. Vertical cross-section of the DDS (bottom left), and the two types of metalization schemes used for the top LED contact (top). The equivalent circuits EC1 and EC2 (bottom right) approximate the electrical response of the DDS mesa. EC1 describes the system in cylindrical coordinates extending ${ }_{85}$ from the mesa center to the mesa edge as illustrated by the dashed lines while EC2 further simplifies the circuit into discrete components. of observing EL cooling at high current injection condition is the generally inefficient light extraction and associated optical losses. The need for electrical injection also introduces several additional nonidealities as compared to the PL cooling. These include the additional optical losses introduced by the electrical contacts as well as the challenges related to efficient excitation of the active region. In particular, in typical devices the current tends to crowd close to the metal electrode reducing the efficiency of the device regions located further from the contact also limiting the device size.

To eliminate some of the key challenges in demonstrating EL cooling, we recently proposed the double diode structure (DDS) framework for studying selected aspects of EL refrigeration mechanisms [13]. As shown in Fig. 1, the DDS is formed of two p-n junctions, operating as an LED and a photodiode (PD), enclosed in a single epitaxially grown semiconductor structure with an effectively homogeneous refractive index. While this specific configuration obviously/lacks the thermal insulation needed between the LED and the PD in practical applications, it nevertheless offers many intriguing properties making it an interesting intermediate research prototype [14]. Once the technical challenges are better understood, it will also be possible to improve the thermal resistance by introducing e.g. vacuum nanogaps or other thermally insulating materials with matched refractive indices within the structure as in the thermophotonic heatpump (THP) configuration [10].

In addition to essentially eliminating the need for light extraction and the directly associated optical losses due to the light trapping in conventional LEDs, the DDS structure has also another significant benefit as it includes a built-in PD which allows an easy way to measure photon and energy transport within the structure. Furthermore, in this letter it is argued that the DDS can even eliminate the adverse effects of limited material conductivity and current crowding in high efficiency devices due to carrier generation in the PD, allowing to fabricate large devices where the light can be efficiently collected by the absorber. In parallel we also demonstrate how current spreading affects light emission uniformity in large area DDSs, and how the current crowding issues gradually disappear as the efficiency of the structure increases.

The epiwafers for the DDSs were grown by metal-organic vapor phase epitaxy (MOVPE) and subsequently processed to define coupled LED and PD mesas with diameters between $100-1000 \mu \mathrm{m}$. The fabrication procedure consists of several consecutive steps of standard optical lithography and selective wet chemical etching steps using thin AlAs layers 
as etch stop layers. The fabrication process is described in more detail in our previous publication

$90 \quad$ [13]. Here we will just briefly mention the differences45 as compared to the previously published process for conciseness. To be able to optically study the spatial distribution of light emission, we fabricated two types of devices: devices with fully metalized top

95 mesa contacts performing as the reference to ensureso that the samples are comparable based on the IV measurements, and devices with top mesa contacts having regularly distributed small circular openings as illustrated in Fig. 1 to sample the optical emission within the device. As compared to the previously55 studied molecular-beam epitaxy (MBE) grown devices [13] the epistructure was the same but in present it does not include omnidirectional reflectors (ODR) on the LEDs top contact. The key device layers in the and the n-type CSL with a thickness of $1600 \mathrm{~nm}$ within the LED mesa and $700 \mathrm{~nm}$ outside the mesa. The other layer dimensions and material compositions are given in our previous publication [13].

The DDSs were analyzed both electrically and65 optically. In general, the DDS is a three terminal device formed of two diodes, with cathodes that are connected together as a common ground terminal as illustrated in Fig.1 (EC2). It should be mentioned

115 that the cathode thickness $(\sim 1 \mu \mathrm{m})$ is much smaller than the mesa diameter $(100-1000 \mu \mathrm{m})$. Measuring the currents through the top (LEDs) and bottom (PDs) terminals allows to determine simultaneously both LED and PD voltages and currents. One of the most

${ }_{120}$ obvious parameters quantifying the energy transfer75 between the emitter and absorber is the coupling quantum efficiency (CQE), which approximates the efficiency of photon transfer between LED and PD. While we define the CQE as $\eta=I_{2} / I_{1}$, where $I_{1}$

125 is the current injected through the LED and $I_{2}$ isoo the photocurrent generated by the PD under short circuit conditions, the electrical behaviour of the PD is expected to change very slowly near the shortcircuit condition. Because the PD current also includes

130 e.g. recombination losses in the absorber, the $\mathrm{CQF}_{85}$ represents a lower bound of photon transport efficiency between emitter and absorber. Due to the definition of the CQE it is also affected by LED efficiency and its dependence on the bias. For imaging the spatial

135 distribution of the light generation in the DDS withoo the perforated top contact a standard CMOS camera was used.

To better understand the electronic behavior of the intracavity configuration, we also simulate ${ }_{140}$ the devices using the drift-diffusion (DD) equation\$95 for electronic charge transport coupled to a first order photon transport model. The DD framework numerically solves for the coupled DD current and continuity equations for charge carriers, by calculating the distributions of the electrostatic potential and the quasi-Fermi levels for electrons and holes in the structure [15]. Electron-hole recombination rates are included using the well-known parameterized formulas for radiative, Shockley-Read-Hall (SRH), Auger recombination [16], and surface and interface non-radiative recombination [17]. We use a radiative recombination constant $B=2 \times 10^{-10}\left(\mathrm{~cm}^{3} \mathrm{~s}^{-1}\right)$ [18] and an Auger recombination constant $C=$ $10^{-30}\left(\mathrm{~cm}^{6} \mathrm{~s}^{-1}\right)[19]$. The combined effect of $\mathrm{SRH}$, surface and interface non-radiative recombination parameters are device dependent and are extracted from measurements for each device. The LED and the photodiode are optically coupled using the BeerLambert law [20] based absorption rate in the PD, with an effective absorption coefficient of $\zeta=10^{4}\left(\mathrm{~cm}^{-1}\right)$ [21] and an optical coupling factor of $90 \%$. Further details about the full 3D simulation model can be found elsewhere $[14,16,22]$.

The full DD model can additionally be approximated by a much simpler equivalent circuit model EC1 of Fig. 1 described by the drift and current continuity equations $d U(r) / d r=\rho I(r) / r$ and $d I(r) / d r=$ $q r[R(r)-G(r)]=q r(1-\eta) R(r)$, where $U(r)$ is the voltage over the distributed LED, $I(r)$ is the cylindrical/current towards the mesa center in the current spreading layer (CSL), $\rho$ is the effective sheet resistivity of the CSL, and the cylindrical symmetry of the structure is accounted for by the specific inclusion of the radial coordinate $r$. The recombination in the LED follows the simplified exponential law $R=R_{0} e^{q U / k T}$, and the generation $G(r)$ in the PD is coupled to the recombination by the factor $\eta$ corresponding here to CQE. With the boundary conditions at the mesa center $I(0)=0$, the solution of these equations is $I(r)=I(L) r^{2} /\left[\sigma I(L)\left(L^{2}-r^{2}\right)+L^{2}\right]$ and $R(r)=d I(r) /[q r(1-\eta) d r]$, where the current $I(L)$ at the mesa edge is $I(L)=I_{1}(1-\eta), I_{1}$ is the total current injected to the LED through the top contact and $\sigma=q \rho /\left(4 k_{b} T\right)$. To obtain a better understanding of the resulting nonuniformity of the recombination, we can use the analytical solutions to calculate the quantity $R(L) / R(0)$, i.e. the ratio of the recombination rate at the mesa edge to the recombination rate at the mesa center. This results in $R(L) / R(0)=\left[\sigma I_{1}(1-\eta)+1\right]^{2}$, showing that the recombination is most uniform for small currents or near unity CQE. The spatial characteristics of the recombination will be investigated in more detail in the context of Fig. 4 towards the end of the paper.

The typical $I-V$ curves of the DDS showing the current through the LED and the PD of a fully metalized DDS, as a function of the emitter voltage, are 

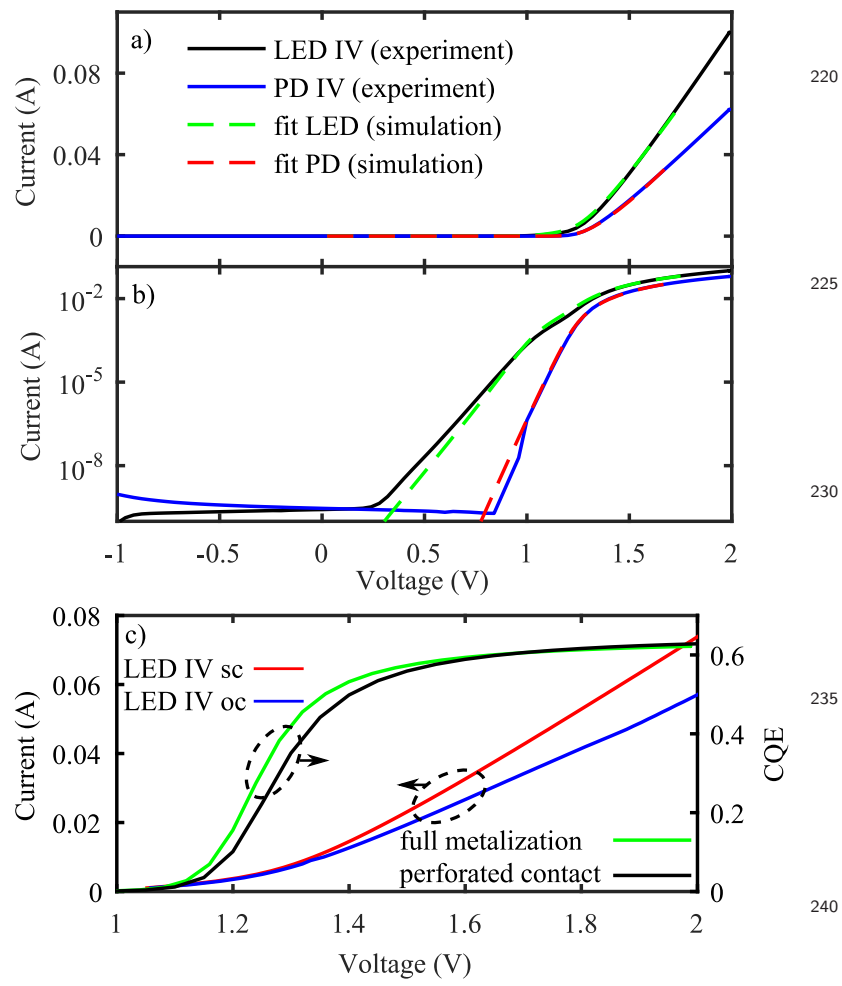

Figure 2. Typical experimentally measured $I-V$ curves showing the current through the LED and PD as a function of voltage between the LED and common terminals for a $\mathrm{DDS}_{45}$ with a $500 \mu \mathrm{m}$ diameter and full metalization on a linear (a) and a semilogarithmic scale (b). The dashed lines show the corresponding results from the simulations. Experimental dependence of (left axis) the LED current on the voltage between the LED and common terminals when the PD of the $500 \mu \mathrm{m}$ diameter DDS with perforated contact is open (oc) or short (sc) circuited, and (right axis) the corresponding CQE for the same DDSs (c).

presented in Fig. 2a-b. It can be seen that at currents exceeding $\sim 10 \mathrm{~mA}$ (on average $\sim 5 \mathrm{~A} / \mathrm{cm}^{2}$ ), electrical ${ }_{55}$ characteristics of both diodes are mainly determined by the internal resistance of the LED. Ideality factors extracted from the exponential portion of the $I-V$ curves are 2 and 1 for LED and PD respectively. These values show that at reasonably low biases, the $I-V_{260}$ 205 behavior of the LED is dominated by Shockley-ReadHall (SRH) -like recombination, while the bimolecular form of the $\mathrm{PD}$ current shows that the radiative recombination in the $\mathrm{LED}$ is also of the bimolecular form. To ensure that the measurements and diode $\mathrm{E}_{65}$ ${ }_{210}$ behavior is not significantly affected by thermal effects at the higher end of current densities, we carried out the measurements both under pulsed (1\% duty cycle) and continuous excitation conditions. We also simulated the temperature profile in the device using 215 coupled DD and heat diffusion equations, finding that even at the highest injection currents the temperature in the device only rises by $\sim 10 \mathrm{~K}$ at the edge of the top contact and far less elsewhere. The dashed lines show the corresponding results from the full DD simulations.

The DDS with openings in the top contact shows very similar $I-V$ behavior with notable differences only in their ideality factors in the $\mathrm{SRH}$ dominated regime where the LED ideality factor is closer to 1.8 . The most plausible reason for this difference is that the 225 removal of the metal contact leads to stronger Fermilevel pinning on the surface and thereby makes current spreading in the opening less efficient. However, the general behavior of $I-V$ curves as well as the CQE, presented in Fig. 2c, are essentially unchanged

230 for the DDS with openings and the reference DDS with fully metalized contact. The CQE for both structures peaks at $63 \%$ improving the previously reported value of $53 \%$ by about $10 \%$ [13]. Similarity of the electrical characteristics (as well as any simple reflectivity estimates) also indicates negligible light extraction out of the holes in top contact, which allows to use the structure with openings for all further analysis.

The EC model and simulations suggest that the photogenerated carriers in the PD play an important role in determining the effective conductivity of the current spreading layer of the DDS. Fig. 2c also showsthe $I-V /$ characteristics of the LED with openings under two distinct conditions. In the opencircuit (oc) condition there is no current flow through the absorber, which emulates a DDS with $\eta=0$. This forces the photogenerated current to be zero and guides all the LED current through the common terminal. In contrast, short-circuiting (sc) the PD will lead to the largest photogenerated current with a smaller fraction of the current going to the common CSL. For the sc conditions the total current through the LED for a given bias voltage is significantly larger than in the oc case. This difference can be qualitatively explained using the simplified equivalent circuit EC2 of Fig 1. The current in the LED terminal for the sc case can be expressed as $I_{1}=I_{2}+I_{\text {common }}=\eta I_{1}+I_{\text {common }}$ where the notation of the currents corresponds to the notation in Fig. 1. In terms of the voltage loss in the resistor $R$ in EC2 this implies $\Delta U_{s c}=R I_{1}(1-\eta)$. In the oc case, where no current flows through the PD, the voltage drop is similarly $\Delta U_{o c}=R I_{1}$. This shows that for the oc conditions the resistive loss in resistor $R$ is substantially higher than for the sc, suggesting that the macroscopic $I-V$ behaviour of the system changes. To understand the reasons for this we analyze the spatial distribution of the recombination in DDS with perforated top contact.

To analyze the distribution of the light generation over the whole DDS area we made microphotographs of the LED luminescence through the openings in the top contact. Fig. 3a shows the distribution of the LED emission intensity imaged through the openings 

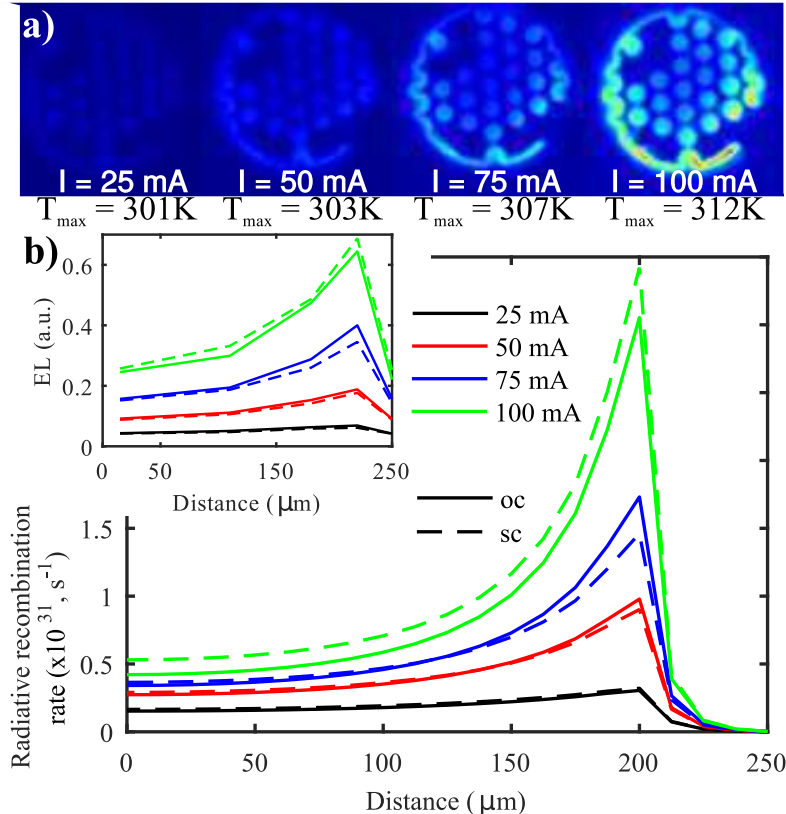

Figure 3. a) Mapping of the LED emission at different excitation currents for $500 \mu \mathrm{m}$ DDS size with PD under opencircuit condition. Temperature values represent peak values estimated from the simulations within the DD model coupled with the heat diffusion equation under steady state curren $\mathbf{k}^{10}$ injection. b) Radial distribution of the recombination rate in the DDS LED from simulation. Inset: experimental radial distribution of EL as measured through the contact openings.

as a function of the LED current, also marking the $\mathrm{e}^{15}$ estimated peak temperatures occurring under the edge of p-type contact as obtained from the numerical simulations for different bias conditions. In the inset, the intensity has been further processed to show the average intensity through each opening as a function ${ }^{20}$ of the distance from the mesa center. Especially for the high current densities the EL is not uniform but exhibits much stronger emission at the edge than in the center. This is in a good agreement with simulated radiative recombination rates shown in Fig?25

$2853 \mathrm{~b}$. Also, in analogy with the EC models, the spatial distribution of the recombination rates is affected by short- or open-circuiting the PD terminals and for all studied cases the emission is more uniform when the $\mathrm{PD}$ is short-circuited. The dependence of the net rate ${ }^{30}$ of radiative recombination on the PD bias is simply caused by plotting the figures for a fixed total current leading to different bias voltages needed to drive the current. This leads to different LED efficiencies and different values of net radiative recombination rates.35 295 Effectively, this improved uniformity of the sc case indicates that radiative recombination will be more uniform for higher DDS efficiencies.

The general effect of the changes in the CQE as obtained from the EC model and DD simulation $3^{40}$ 300 is summarized in Fig. 4, which shows the spatial

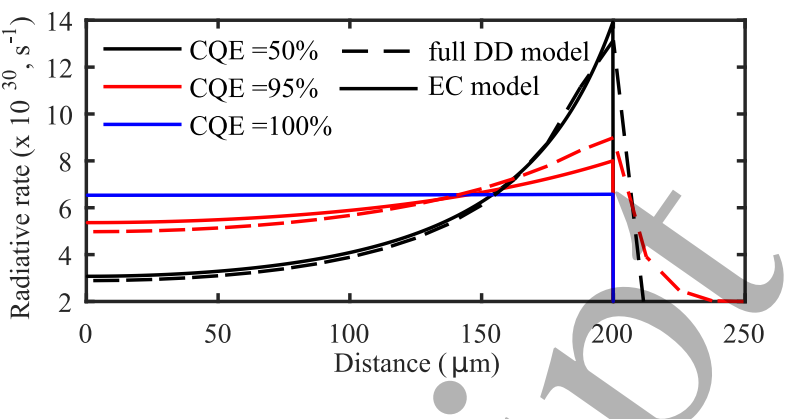

Figure 4. Comparison of recombination rates in LED active region at different CQEs obtained from DD and EC models for a total LED current of $50 \mathrm{~mA}\left(25.4 \mathrm{~A} / \mathrm{cm}^{2}\right)$.

distribution of the radiative recombination in the LED as a function of the radial position for selected values of the CQE and total LED current of $50 \mathrm{~mA}$. The results show a good agreement between the DD simulations and the analytic EC model, both predicting that as the CQE increases the recombination profile becomes flatter and completely evens out at the limit CQE $\rightarrow$ 1. At this limit there will be no lateral current flowing in the CSL. Combinination of the facts that improved CQE leads to more uniform light emission with the well known result that homogeneous excitation results in a larger average efficiency of light emission [23] indicates that the current crowding and the CQE exhibit a mutual dependence where improving one also improves the other in a coupled fashion. If sufficiently strong, this dependence may substantially facilitate the process of maximizing the DDS CQE.

In conclusion, we analyzed the effect of current spreading on the spatial uniformity of light emission in the DDS using simulations and optical imaging techniques while electrically limiting the photogenerated current passing through the device. We found that the photogenerated current in the PD substantially reduces the lateral current in the current spreading layer and thereby reduces the associated electrical current spreading losses, enabling more uniform current spreading and light emission in the LED. For sufficiently high CQE, this removes one of the bottlenecks in using large area devices in the study of EL cooling, allowing the design of devices with smaller fraction of nonradiative recombination and uncontrolled optical emission through the edges of the device. The studied DDS also demonstrated a 10\% improvement for the CQE, peaking at $63 \%$. In addition, at the limit of a high CQE the lateral current starts to diminish quickly, leading to a mutual dependence between the CQE and light emission distribution. This is expected to facilitate the remaining steps in optimizing the DDS towards the performance needed to demonstrate EL cooling. 
This project has received funding from the Academy of Finland and the European Research Council (ERC) under the European Unions Horizon 3452020 research and innovation programme (grant agreement No 638173). We acknowledge the provision of facilities and technical support from Micronova Nanofabrication Centre at Aalto University. We also thank Dr. Wlodek Strupinski from Institute of 350 Electronic Materials Technology, Warsaw, Poland, for providing us with epitaxial materials.

[1] Keyes R J and Quist T M 1962 Proceedings of the IRE 50 $1822-1823$

355 [2] Tauc J 1957 Czechoslovak Journal of Physics 7 275-276

[3] Heikkilä O, Oksanen J and Tulkki J 2009 Journal of Applied Physics 105093119

[4] Santhanam P, Gray D J and Ram R J 2012 Physical Review Letters 108

360 [5] Xue J, Li Z and Ram R J 2017 Physical Review Applied 8

[6] Sheik-Bahae M and Epstein R I 2007 Nature Photonics 1 $693-699$

[7] Xue J, Zhao Y, Oh S H, Herrington W F, Speck J S, DenBaars S P, Nakamura S and Ram R J 2015 Applied

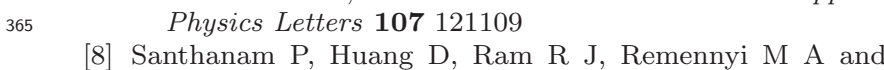
Matveev B A 2013 Applied Physics Letters 103183513

[9] Santhanam P, Huang D, Gray D J and Ram R J 2013 Electro-luminescent cooling: light emitting diodes above unity efficiency ed Epstein R I, Seletskiy D V and SheikBahae M p 863807

[10] Oksanen J and Tulkki J 2010 Journal of Applied Physics 107093106 (7 p.)

[11] Chen K, Xiao T P, Santhanam P, Yablonovitch E and Fán S 2017 Journal of Applied Physics 122143104

375

[12] Tiira J, Radevici I, Haggren T, Hakkarainen T, Kivisaari P, Lyytikainen J, Aho A, Tukiainen A, Guina M and Oksanen J 2017 Intracavity double diode structures with GaInP barrier layers for thermophotonic cooling ed

380 Epstein R I, Seletskiy D V and Sheik-Bahae-M p 1012109

[13] Olsson A, Tiira J, Partanen M, Hakkarainen T, Koivusalo E, Tukiainen A, Guina M and Oksanen J 2016 IEEE Transactions on Electron Devices 63 3567-3573

[14] Sadi T, Kivisaari P, Tiira J, Radevici I, Haggren T and Oksanen J 2018 Optical and Quantum Electronics 50

385

[15] Sadi T, Kivisaari P, Tiira J, Radevici I, Haggren T and Oksanen J 2017 Modeling of charge and photon transport in coupled intracavity light emitters ed Piprek $\mathrm{J}$ and Willatzen M (In Proc. International Conference

390 on Numerical Simulation of Optoelectronic Devices (NUSOD)) pp 201-202

[16] Kivisaari P, Oksanen J, Tulkki J and Sadi T 2015 Journal of Computational Electronics 14 382-397

[17] Chen Y, Kivisaari P, Pistol M E and Anttu N 2016 Nanotechnology 27435404

395

[18] Varshni Y P 1967 phys. stat. sol. (b) 19 459-514

[19] Strauss U, Rühle W W and Köhler K 1993 Appl. Phys. Lett. $6255-57$

400

[20] Mayerhöfer T G, Mutschke H and Popp J 2016 ChemPhysChem 17 1948-1955

[21] Casey H C, Sell D D and Wecht KW 1975 J. Appl. Phys. 46 250-257

[22] Kivisaari P, Sadi T, Li J, Rinke P and Oksanen J 2017 Advanced Electronic Materials 31600494

405 [23] Liancheng Wang, Zi-Hui Zhang and Ning Wang 2015 IEEE Journal of Quantum Electronics 51 1-9

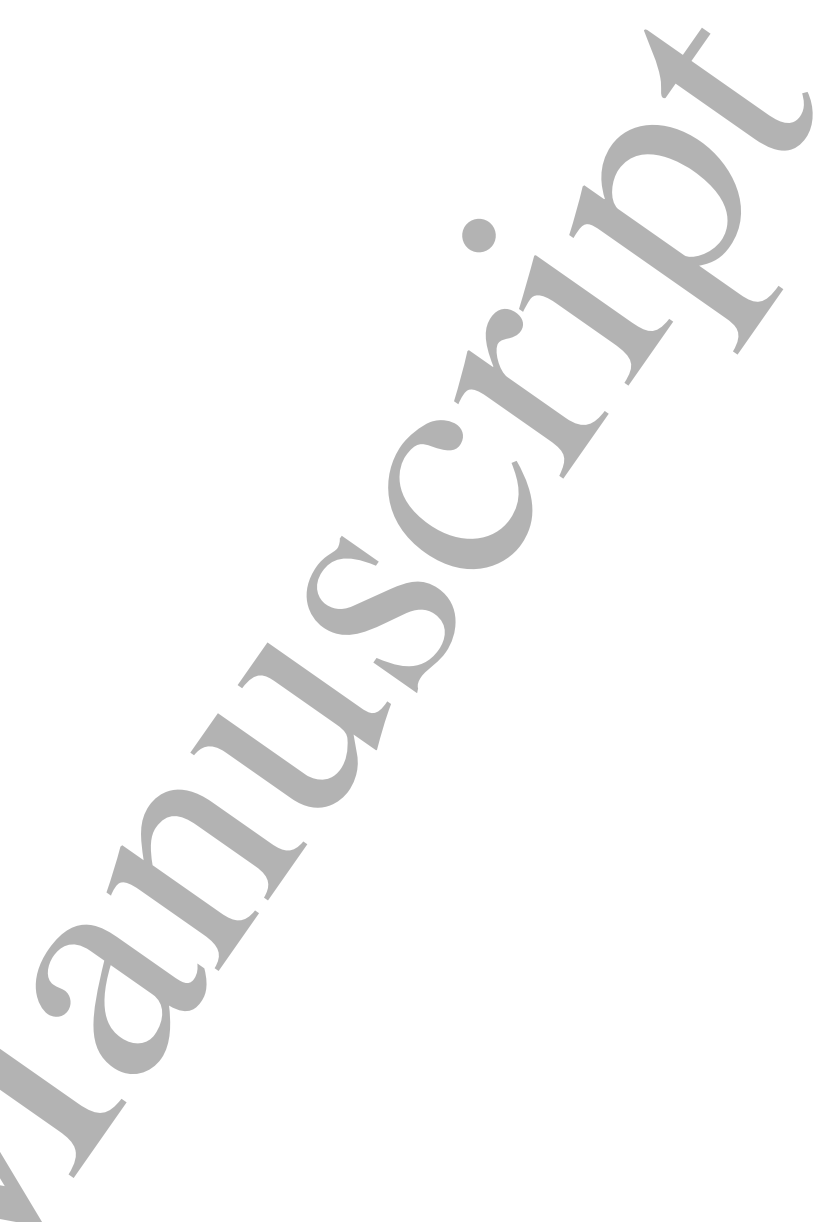

